Commun. Fac. Sci. Univ. Ank. Series $A_{1}$

V. 38, Number 1-2, pp. 77-86 (1989)

\title{
THE RELATION AMONG BLASCHKE VECTORS OF RULED SURFACES ON A LINE CONGRUENCE AND ITS CONSEOUENCES
}

\author{
ALt ÇALIŞKAN
}

E.Ü. Fen Fakültesi Matematik Bölümü, Izmir-Turkey

\section{ABSTRACT}

This study consists of three sections and first of them is reserved for the basic concepts. Necessary concepts and relations obtained among the magnitudes of a ruled surface $\vec{R}_{1}$ and parameter ruled surfaces (chosen as principal ruled surfaces) $\vec{R}_{11}, \vec{R}_{21}$ of a congruence $\vec{R}(u, v)$ are given in the section II so as to use in the third section.

In the section three, the relation

$$
\overrightarrow{\mathrm{B}}=\mathrm{P}\left(\frac{\operatorname{Cos} \psi}{\mathrm{P}} \overrightarrow{\mathrm{B}}_{1}+\frac{\operatorname{Sin} \psi}{\mathrm{P}_{2}} \overrightarrow{\mathrm{B}}_{2}+\frac{\mathrm{d} \psi}{\mathrm{dS}} \overrightarrow{\mathbf{R}}_{\mathrm{o}}\right)
$$

is found among the Blaschke vectors (dual instantaneous speeds) of the three ruled surfaces mentioned in the sectin II. Using this relation, the formula of Liouville, J. for the theory of surfaces is generalized to the line space by giving the relation among the dual spherical curvaturs which are the invariants of the ruled surfaces, and some corollaries concerning with theme relations are given.

\section{Basic Concepts}

I.1. A ruled surface $\overrightarrow{\mathbf{R}}_{1}$ is given by a unit dual vector depending on one real parameter $t: \vec{R}_{1}(t)=\vec{r}_{1}(t)+\varepsilon \overrightarrow{\mathbf{r}}_{1}(t)$. One of the invarints of this ruled surface is

$$
\Sigma=\frac{Q}{P}
$$

which is known as dual spherical curvature of the ruled surface, (3).

The relation

$$
<\overrightarrow{\mathbf{R}}_{\mathrm{u}}, \overrightarrow{\mathrm{R}}_{\mathrm{v}}>=\mathrm{F}=\mathrm{f}+\varepsilon \overline{\mathbf{f}}=0
$$

ISSN 02571-081 A.Ü. Basimevi 
characterizes that principal ruled surfaces are being taken as parameter ruled surfaces for a line congruence which is given by a dual vector depending on two real parameter:

$$
\overrightarrow{\mathbf{R}}(\mathrm{u}, \mathrm{v})=\overrightarrow{\mathbf{r}}(\mathrm{u}, \mathrm{v})+\varepsilon \overrightarrow{\mathbf{r}}(\mathrm{u}, \mathrm{v})
$$

,(2).

I.2. Let $\vec{w}$ be Darboux vector (instantaneous rotational vector) of the solid trihedron $\left(\vec{e}_{1}, \vec{e}_{2}, \vec{e}_{3}\right)$ moving with respect to a real parameter t. Derivative vectors of this trihedron are:

$$
\frac{\overrightarrow{d e}_{i}}{d t}=\vec{W} \wedge \overrightarrow{e_{i}},(i=1,2,3)
$$

$(4,5)$.

\section{Preliminaries}

$A$ ruled surface and parameter ruled surfaces of the congruence (I.3) are given by the unit dual vectors $\vec{R}_{1}=\vec{R}_{1}(t)=\vec{R}(u(t), v(t))$ and $\vec{R}_{11}=\vec{R}_{11}\left(u, v_{0}\right), \vec{R}_{21}=\vec{R}_{21}\left(u_{0}, v\right)$ respectively. Blaschke trihedron of these rules surfaces passing through the common line $\vec{R}_{0}=$ $\vec{R}_{0}\left(u_{0}, v_{0}\right)$, for the value $t=t_{0}$, of the congruence $\vec{R}(u, v)$ are:

$$
\left(\overrightarrow{\mathbf{R}}_{0}=\overrightarrow{\mathbf{R}}_{1}, \overrightarrow{\mathbf{R}}_{2}, \overrightarrow{\mathbf{R}}_{3}\right),\left(\overrightarrow{\mathbf{R}}_{0}=\overrightarrow{\mathbf{R}}_{11}, \overrightarrow{\mathbf{R}}_{12}, \overrightarrow{\mathbf{R}}_{13}\right),\left(\overrightarrow{\mathbf{R}}_{0}=\overrightarrow{\mathbf{R}}_{21}, \overrightarrow{\mathbf{R}}_{22}, \overrightarrow{\mathbf{R}}_{23}\right)
$$

Blaschke vectors of these trihedrons

$$
\overrightarrow{\mathbf{B}}=Q \overrightarrow{\mathbf{R}}_{0}+\mathbf{P} \overrightarrow{\mathbf{R}}_{3}, \overrightarrow{\mathbf{B}}_{1}=Q_{1} \overrightarrow{\mathbf{R}}_{0}+\mathbf{P}_{1} \overrightarrow{\mathbf{R}}_{13}, \overrightarrow{\mathbf{B}}_{2}=\mathbf{Q}_{2} \overrightarrow{\mathbf{R}}_{0}+\mathbf{P}_{2} \overrightarrow{\mathbf{R}}_{23}
$$

and dual arc elements of these ruled surfaces

$$
\mathrm{dS}=\mathrm{Pdt}, \mathrm{dS}_{1}=\mathbf{P}_{1} \mathrm{du}, \mathrm{dS}_{2}=\mathbf{P}_{2} \mathrm{dv}
$$

are given respectively, where

$$
\mathbf{P}=\sqrt{\overrightarrow{\mathbf{R}}_{1}^{\prime 2}}, \mathbf{P}_{1}=\sqrt{\overrightarrow{\mathbf{R}_{\mathrm{u}}^{2}}}=\sqrt{\mathrm{E}}, \mathbf{P}_{2}=\sqrt{\overrightarrow{\mathbf{R}}_{\mathrm{v}}^{2}}=\sqrt{\bar{G}}
$$

When parameter ruled surfaces are taken as principal ruled surfaces, we have 
THE RELATION AMONG BLASCHKE VECTORS ON A LINE CONGR̃UENCE 79

$$
<\overrightarrow{\mathbf{R}}_{12}, \overrightarrow{\mathbf{R}}_{22}>=0
$$

and

$$
\overrightarrow{\mathbf{R}}_{12} \times \overrightarrow{\mathbf{R}}_{22}=\frac{\overrightarrow{\mathbf{R}}_{\mathrm{u}} \times \overrightarrow{\mathbf{R}}_{\mathrm{v}}}{\sqrt{\mathrm{EG}}}=\overrightarrow{\mathbf{R}}_{\mathrm{o}}
$$

If the dual angle between the unit dual vectors $\vec{R}_{2}$ and $\vec{R}_{12}$ is $\psi$, then we have

$$
\begin{gathered}
\overrightarrow{\mathbf{R}}_{2}=\operatorname{Cos} \psi \overrightarrow{\mathbf{R}}_{12}+\operatorname{Sin} \psi \overrightarrow{\mathbf{R}}_{22} \\
\operatorname{Cos} \psi=\frac{\mathrm{dS}_{1}}{\mathrm{dS}}=\sqrt{\mathbf{E}} \frac{\mathbf{d u}}{\mathrm{dS}}, \operatorname{Sin} \psi=\frac{\mathrm{dS}_{2}}{\mathrm{dS}}=\sqrt{\mathrm{G}} \frac{\mathbf{d v}}{\mathrm{dS}}
\end{gathered}
$$

(1).

Theorem II1. The edges of Blaschke trihedrons of parameter ruled surfaces coincide with each other under the condition that their direction and orders are not the same, e.i:

$$
\begin{aligned}
& \overrightarrow{\mathbf{R}}_{13}=\overrightarrow{\mathbf{R}}_{22} \text { ve } \overrightarrow{\mathbf{R}}_{23}=-\overrightarrow{\mathbf{R}}_{12} \\
& \left(\overrightarrow{\mathbf{R}}_{0}=\overrightarrow{\mathbf{R}}_{11}=\overrightarrow{\mathbf{R}}_{21}, \overrightarrow{\mathbf{R}}_{12}=-\overrightarrow{\mathbf{R}}_{23}, \overrightarrow{\mathbf{R}}_{13}=\overrightarrow{\mathbf{R}}_{22}\right)
\end{aligned}
$$

Corollary II.1. The Blaschke vectors (II.2) can be only expressed by the vectors $\overrightarrow{\mathbf{R}}_{12}, \overrightarrow{\mathbf{R}}_{22}$ and $\overrightarrow{\mathbf{R}}_{0}$.

Corollary II.2. The edges of Blaschke trihedron (II.10) change as function of the both parameters $u$ and $v$, and by taking (II.9) into account, we have

$$
\begin{aligned}
& \frac{\partial \overrightarrow{\mathbf{R}}_{0}}{\partial \mathbf{u}}=\overrightarrow{\mathbf{B}}_{1} \times \overrightarrow{\mathbf{R}}_{0}, \frac{\partial \overrightarrow{\mathbf{R}}_{\mathbf{0}}}{\partial \mathbf{v}}=\overrightarrow{\mathbf{B}}_{2} \times \overrightarrow{\mathbf{R}}_{0}, \frac{\partial \overrightarrow{\mathbf{R}}_{12}}{\partial \mathbf{v}}=\overrightarrow{\mathbf{B}}_{2} \times \overrightarrow{\mathbf{R}}_{12} \\
& \frac{\partial \overrightarrow{\mathbf{R}}_{12}}{\partial \mathbf{u}}=\overrightarrow{\mathbf{B}}_{1} \times \overrightarrow{\mathbf{R}}_{12}, \frac{\partial \overrightarrow{\mathbf{R}}_{13}}{\partial \mathbf{u}}=\overrightarrow{\mathbf{B}}_{1} \times \overrightarrow{\mathbf{R}}_{13}, \frac{\partial \overrightarrow{\mathbf{R}}_{13}}{\partial \mathbf{v}}=\overrightarrow{\mathbf{B}}_{2} \times \overrightarrow{\mathbf{R}}_{13} \text { (II.11) } \\
& \frac{\partial \overrightarrow{\mathbf{R}}_{22}}{\partial \mathbf{u}}=\overrightarrow{\mathbf{B}}_{1} \times \overrightarrow{\mathbf{R}}_{22}, \frac{\partial \overrightarrow{\mathbf{R}}_{22}}{\partial \mathbf{v}}=\overrightarrow{\mathbf{B}}_{2} \times \overrightarrow{\mathbf{R}}_{22}, \frac{\partial \overrightarrow{\mathbf{R}}_{23}}{\partial \mathbf{u}}=\overrightarrow{\mathbf{B}}_{1} \times \overrightarrow{\mathbf{R}}_{23}, \frac{\partial \overrightarrow{\mathbf{R}}_{23}}{\partial \mathbf{v}}= \\
& \overrightarrow{\mathbf{B}}_{2} \times \overrightarrow{\mathbf{R}}_{23}
\end{aligned}
$$


Corollary II.3. For the parameter ruled surfaces $\vec{R}_{11}$ and $\vec{R}_{22}$ we have.

$$
\begin{aligned}
& <\mathbf{R}_{12}, \frac{\partial \overrightarrow{\mathbf{R}}_{22}}{\partial \mathbf{S}_{1}}>=-<\overrightarrow{\mathbf{R}}_{22}, \frac{\partial \overrightarrow{\mathbf{R}}_{12}}{\partial \mathbf{S}_{1}}>=\frac{(\sqrt{\mathbf{E}) \mathbf{v}}}{\sqrt{\mathbf{E G}}} \\
& <\overrightarrow{\mathbf{R}}_{22}, \frac{\partial \overrightarrow{\mathbf{R}}_{12}}{\partial \mathbf{S}_{2}}>=-<\overrightarrow{\mathbf{R}}_{12}, \frac{\partial \overrightarrow{\mathbf{R}}_{22}}{\partial \mathbf{S}_{2}}>=\frac{(\sqrt{\mathbf{G}) \mathbf{u}}}{\sqrt{\mathbf{E} \mathbf{G}}}
\end{aligned}
$$

(1).

III. The relation among Blaschke vectors of ruled surfaces of a line congruence (in this section, parameter ruled sufaces are taken as principal ruled surfaces)

Theorem III.1. Let $\overrightarrow{\mathbf{R}}_{1}$ and $\overrightarrow{\mathbf{R}}_{11}, \overrightarrow{\mathbf{R}}_{21}$ be a ruled surface and parameter ruled surfaces of a congruence $\vec{R}(u, v)$ recpectively. $P_{1}$ and $\mathbf{P}_{2}$ be magnitudes, $\vec{B}_{1}$ and $\vec{B}_{2}$ be Blaschke vectors of the parameter ruled surfaces $\vec{R}_{11}$ and $\vec{R}_{21}$ respectively. Then we have

$$
\frac{d \overrightarrow{\mathbf{R}}_{12}}{\mathrm{dS}}=\overrightarrow{\mathrm{C}} \times \overrightarrow{\mathbf{R}}_{12}, \frac{\mathrm{d} \overrightarrow{\mathbf{R}}_{22}}{\mathrm{dS}}=\overrightarrow{\mathrm{C}} \times \overrightarrow{\mathbf{R}}_{22}, \frac{\mathrm{d} \overrightarrow{\mathbf{R}}_{0}}{\mathrm{dS}}=\overrightarrow{\mathbf{C}} \times \overrightarrow{\mathbf{R}}_{0}
$$

, where $\psi$ is the dual angle between the unit dual vectors $\vec{R}_{2}$ and $\overrightarrow{\mathbf{R}}_{12}$, which are the second edges of Blaschke trihedrons of the ruled surfaces $\vec{R}_{1}$ and $\vec{R}_{11}$, and where

$$
\overrightarrow{\mathrm{C}}=\frac{\operatorname{Cos} \psi}{\mathrm{P}_{1}} \vec{B}_{1}+\frac{\operatorname{Sin} \psi}{\mathbf{P}_{2}} \vec{B}_{2}
$$

Proof: Since (II.12) and (II.7), the unit dual vectors $\vec{R}_{12}, \vec{R}_{22}$ and $\vec{R}_{2}$ can be expressed as the functions of dual arc lengts $S_{1}$ and $S_{2}$, By using the two last relations of (II.3) and (II.4), and the relations (II.8), (II.11) can be written as the following: 
THE RELATION AMONG BLASCHKE VECTORS ON A LINE CONGRUENCE 81

$$
\begin{aligned}
\frac{\mathbf{d R}_{12}}{\mathrm{dS}}=\frac{\partial \overrightarrow{\mathbf{R}}_{10}}{\partial \mathrm{S}_{1}} \frac{\mathrm{d} \mathrm{S}_{1}}{\mathrm{dS}}+\frac{\partial \overrightarrow{\mathbf{R}}_{12}}{\partial \mathrm{S}_{2}} \frac{\partial \mathrm{S}_{2}}{\partial \mathrm{S}} & =\overrightarrow{\mathbf{B}}_{1} \times \overrightarrow{\mathbf{R}}_{12} \frac{\operatorname{Cos} \psi}{\sqrt{\mathbf{E}}} \\
& +\overrightarrow{\mathbf{B}}_{2} \times \overrightarrow{\mathbf{R}}_{12} \frac{\operatorname{Sin} \psi}{\sqrt{\mathbf{G}}}
\end{aligned}
$$

If we consider (III.1) and (II.4), we have

$$
\frac{\mathrm{d} \overrightarrow{\mathbf{R}}_{12}}{\mathrm{dS}}=\overrightarrow{\mathrm{C}} \times \overrightarrow{\mathbf{R}}_{12}
$$

and using the similar way, we have

$$
\frac{d \vec{R}_{22}}{d S}=\vec{C} \times \vec{R}_{22}
$$

from the relation (II.6)

$$
\frac{d \vec{R}_{o}}{d \mathbf{S}}=\frac{d}{d S}\left(\vec{R}_{12} \times \vec{R}_{22}\right)=\frac{d \vec{R}_{12}}{d S} \times \vec{R}_{22}+\vec{R}_{12} \times \frac{d \vec{R}_{22}}{d S}
$$

and if we use the relations (III.3,4,5 and 6)

$\frac{d \overrightarrow{\mathbf{R}}_{\mathbf{O}}}{\mathbf{d S}}=\left(\overrightarrow{\mathrm{C}} \times \overrightarrow{\mathbf{R}}_{12}\right) \times \overrightarrow{\mathbf{R}}_{22}+\overrightarrow{\mathbf{R}}_{12} \times\left(\overrightarrow{\mathrm{C}} \times \overrightarrow{\mathbf{R}}_{22}\right)=\overrightarrow{\mathrm{C}} \times\left(\overrightarrow{\mathbf{R}}_{12} \times \overrightarrow{\mathbf{R}}_{22}\right)$

and by taking (II.6) into account, we have

$$
\frac{d \overrightarrow{\mathbf{R}}_{\mathbf{o}}}{\mathrm{d} \mathrm{S}}=\overrightarrow{\mathbf{C}} \times \overrightarrow{\mathbf{R}}_{\mathbf{o}}
$$

And from the relations (III.3), (III.4) and (III.5) we get the rela:ion (III.2).

Corollary III.1. For the parameter ruled surfaces $\overrightarrow{\mathbf{R}}_{11}$ and $\overrightarrow{\mathbf{R}}_{21}$, we write

$$
<\overrightarrow{\mathbf{R}}_{22}, \frac{\mathbf{d} \overrightarrow{\mathbf{R}}_{12}}{\mathbf{d S}}>=-<\overrightarrow{\mathbf{R}}_{12}, \frac{d \overrightarrow{\mathbf{R}}_{22}}{\mathbf{d S}}>=<\overrightarrow{\mathbf{C}}, \quad \overrightarrow{\mathbf{R}}_{\mathbf{0}}>
$$


Proof: From (III.1) and (II.6) $<\overrightarrow{\mathbf{R}}_{22}, \frac{d \overrightarrow{\mathbf{R}}_{12}}{\mathrm{dS}}>=<\overrightarrow{\mathbf{R}}_{22}, \overrightarrow{\mathrm{C}} \times \overrightarrow{\mathbf{R}}_{12}>=<\overrightarrow{\mathbf{R}}_{12} \times \overrightarrow{\mathbf{R}}_{22}, \overrightarrow{\mathrm{C}}>=<\overrightarrow{\mathbf{R}}_{0}, \overrightarrow{\mathrm{C}}_{>}$ and if (II.5) is derived with respect to $S$

$$
<\overrightarrow{\mathbf{R}}_{22}, \frac{\mathrm{d} \overrightarrow{\mathbf{R}}_{12}}{\mathrm{~d} \mathbf{S}}>=-<\overrightarrow{\mathbf{R}}_{12}, \frac{\mathrm{d} \overrightarrow{\mathbf{R}}_{22}}{\mathrm{~d} \mathbf{S}}>
$$

is obtained.

Thearem III.2. Let a ruled surface $\vec{R}_{1}$ and parameter ruled surfaces $\vec{R}_{11}, \vec{R}_{21}$ of a congruence $\vec{R}(u, v)$ pass through the line $\vec{R}_{0}$ of the congruence. Let $\vec{B}$ and $\vec{B}_{1}, \vec{B}_{2}$ be Blaschke vectors of these ruled surfaces respectively, and $\psi$ be angle between the edges $\vec{R}_{2}$ and $\vec{R}_{12}$ of Blaschke trihedrons of the ruled surfaces $\vec{R}_{1}$ and $\vec{R}_{11}$ respectively. Among the Blaschke vectors and the common line $\overrightarrow{\mathbf{R}}_{0}$ of these three ruled surfaces, there exists the relation

$$
\overrightarrow{\mathbf{B}}=\mathbf{P}\left(\frac{\operatorname{Cos} \psi}{\overrightarrow{\mathbf{P}}_{1}} \overrightarrow{\mathbf{B}}_{1}+\frac{\operatorname{Sin} \psi}{\mathbf{P}_{2}} \overrightarrow{\mathbf{B}}_{2}+\frac{\mathrm{d} \psi}{\mathrm{dS}} \overrightarrow{\mathbf{R}}_{0}\right)
$$

,where $P, P_{1}=\sqrt{E}, P_{2}=\sqrt{G}$ are the magnitudes of the ruled surfaces $\vec{R}_{1}, \vec{R}_{11}$ and $\vec{R}_{21}$ respectively, and $d S$ is the dual arc element of the ruled surface $\overrightarrow{\mathbf{R}}_{1}$

Proof: If we take (I.4) and (II.4) into account, since the Blaschke vector of the trihedron $\left(\overrightarrow{\mathbf{R}}_{0}=\overrightarrow{\mathbf{R}}_{1}, \overrightarrow{\mathbf{R}}_{2}, \overrightarrow{\mathbf{R}}_{3}\right)$ is $\overrightarrow{\mathbf{B}}$, we have

$$
\frac{\mathrm{d} \overrightarrow{\mathbf{R}}_{2}}{\mathrm{dS}}=\frac{\mathrm{d} \overrightarrow{\mathbf{R}}_{2}}{\mathrm{dt}} \cdot \frac{1}{\mathbf{P}}=\frac{\overrightarrow{\mathbf{B}} \times \overrightarrow{\mathbf{R}}_{2}}{\mathbf{P}}=\frac{\overrightarrow{\mathbf{B}} \times \overrightarrow{\mathbf{R}}_{0}}{\mathbf{P}}
$$

If (II.7) is derived with respect to $S$ and the two last trihedrons of (II.1) is taken into account and using the relation (II.9), we have 
$\frac{\mathbf{d} \overrightarrow{\mathbf{R}}_{2}}{\mathrm{dS}}=\frac{\mathrm{d} \overrightarrow{\mathbf{R}}_{12}}{\mathbf{d S}} \operatorname{Cos} \psi+\frac{\mathrm{d} \overrightarrow{\mathbf{R}}_{22}}{\mathbf{d S}} \operatorname{Sin} \psi+\overrightarrow{\mathbf{R}}_{\mathbf{0}} \times\left(\overrightarrow{\mathbf{R}}_{22} \operatorname{Sin} \psi+\overrightarrow{\mathbf{R}}_{12} \operatorname{Cos} \psi\right) \frac{\mathrm{d} \psi}{\mathrm{dS}}$ From the relations (III.1), (III.2) and (II.7) we get

$$
\frac{d \overrightarrow{\mathbf{R}}_{2}}{\mathrm{~d} \mathrm{~S}}=\overrightarrow{\mathbf{C}} \times \overrightarrow{\mathbf{R}}_{2}+\left(\overrightarrow{\mathbf{R}}_{0} \times \overrightarrow{\mathbf{R}}_{2}\right) \frac{\mathbf{d} \psi}{d S}=(\overbrace{\vec{C}}+\overrightarrow{\mathbf{R}}_{0} \frac{\overrightarrow{\mathbf{M}} \psi}{d S}) \times \overrightarrow{\mathbf{R}}_{2}
$$

Let the expression in the paranthesis will be denoted by $M$ for shortness then

$$
\overrightarrow{\mathrm{C}}=\overrightarrow{\mathbf{M}}-\overrightarrow{\mathbf{R}}_{\mathrm{o}} \frac{\mathrm{d} \psi}{\mathrm{dS}}
$$

From the two last relations

$$
\frac{d \overrightarrow{\mathbf{R}}_{2}}{\mathbf{d S}}=\overrightarrow{\mathbf{M}} \times \overrightarrow{\mathrm{R}}_{2}
$$

is found. From (III.1) and (III.9)

$$
\frac{\mathrm{d} \overrightarrow{\mathbf{R}}_{\mathrm{o}}}{\mathrm{dS}}=\overrightarrow{\mathbf{M}} \times \overrightarrow{\mathbf{R}}_{\mathbf{o}}
$$

If we compare the relations (III.8) with the relations (III.10) and (III.11)

$$
\overrightarrow{0}=\left(-\overrightarrow{\mathbf{m}}+\frac{\overrightarrow{\mathbf{B}}}{\mathbf{P}}\right) \times \overrightarrow{\mathbf{R}}_{2} ; \overrightarrow{0}=\left(-\overrightarrow{\mathbf{M}}+\frac{\overrightarrow{\mathbf{B}}}{\mathbf{P}}\right) \times \overrightarrow{\mathbf{R}}_{0}
$$

are obtained respectively. From these two relations

$$
\mathrm{U} \overrightarrow{\mathbf{R}}=\mathrm{V} \overrightarrow{\mathbf{R}}_{\mathbf{0}}
$$

can be obtained, where $U$ and $V$ are dual scalars. But the equation (III.12) is not possible due to the first relation of (II.1), thus

$$
\overrightarrow{\mathbf{M}}-\frac{\overrightarrow{\mathrm{B}}}{\mathrm{P}}=\overrightarrow{0} \Rightarrow \overrightarrow{\mathrm{B}}=\mathrm{P} \overrightarrow{\mathrm{M}}
$$


From this relation and (III.9)

$$
\overrightarrow{\mathbf{B}}=\mathbf{P}\left(\overrightarrow{\mathbf{C}}+\overrightarrow{\mathbf{R}}_{\mathrm{o}} \frac{\mathrm{d} \psi}{\mathrm{dS}}\right)
$$

is obtained and by substituting (III.2) in (III.13) we get the relation (III.7)

Depending on the theorem (III.3), the following corollaries can be expressed:

Corollarıy III.2. The Blaschke vector of the thiredron $\left(\overrightarrow{\mathbf{R}}_{1}, \overrightarrow{\mathbf{R}}_{2}\right.$, $\vec{R}_{3}$ ) of $\vec{R}_{1}$ ruled surface of $\vec{R}$ (u.v) congruence can be expressed by the relation (III.13), depending on the Blaschke vector $\vec{C}$ of the trihedron $\left(\overrightarrow{\mathbf{R}}_{1}, \overrightarrow{\mathbf{R}}_{12}, \overrightarrow{\mathbf{R}}_{22}\right)$.

Corollary III.3. We have the relation

$$
\Sigma=\Sigma_{1} \operatorname{Cos} \psi+\Sigma_{2} \operatorname{Sin} \psi+\frac{d \psi}{d S}
$$

Where $\Sigma$ and $\Sigma_{1}, \Sigma_{2}$ are the dual spherical curvatures of a ruled surface and parameter ruled surfaces of a congruence respectively.

Proof: Substituting the Blaschke vectors $\overrightarrow{\mathbf{B}}, \vec{B}_{1}$ and $\vec{B}_{2}$ in (III.7) and expressing dot product of the two side of the equality by $\vec{R}_{0}$

$$
\mathbf{Q}=\mathbf{P}\left(\frac{\operatorname{Cos} \psi}{\mathbf{P}_{1}} \mathrm{Q}_{1}+\frac{\operatorname{Sin} \psi}{\mathbf{P}_{2}} \mathrm{Q}_{2}+\frac{\mathrm{d} \psi}{\mathbf{d S}}\right)
$$

is obtained. Taking (I.1) into consideration, from the relation above we get (III.14). The relation (III.14) is respond to Liouville's formula of the theory of surfaces (III), (VI).

Corollary III.4. For the dual spherical curvature of the ruled surface $\vec{R}_{1}$, we have the relation:

$$
\Sigma=\frac{\mathbf{Q}}{\mathbf{P}}=-<\overrightarrow{\mathbf{R}}_{12}, \frac{\mathrm{d} \overrightarrow{\mathbf{R}}_{22}}{\mathrm{dS}}>+\frac{\mathrm{d} \psi}{\mathrm{dS}}
$$


Proof: From the first relation of (II.2), and (III.13)

$$
\mathbf{Q}=<\overrightarrow{\mathbf{R}}_{0}, \overrightarrow{\mathbf{B}}<=\mathbf{P}<\overrightarrow{\mathbf{R}}_{0}, \overrightarrow{\mathbf{C}}>+\mathbf{P} \frac{\mathrm{d} \psi}{\mathrm{d} S}
$$

By substituting (III.6) in the above equation and reminding

$$
\Sigma=\frac{\mathbf{Q}}{\mathbf{P}} \text {, we get (III.15) }
$$

OZET

Ựç kısımdan oluşan bu çalışmanın birinci kısmı temel bilgilere ayrılmıştır. İkinci kısımda bir $\vec{R}(u, v)$ doğru kongrüansının bir $\vec{R}_{1}$ regle yüzeyi ve asal rengle yüzeyler olarak seçilen $\vec{R}_{11}, \vec{R}_{21}$ parametre regle yüzeylerine ait büyüklükler arasında elde edilmiş olan bilgi ve bağıntılardan üçüneü kısım için gerekli olanlanı verilmişstir.

Ų̛̣üncï kısımda, kısım ikide konu edilen üç regle yüzeyin Blaschke vektörleri (dual ani hizları) arasında

$$
\overrightarrow{\mathbf{B}}=\mathbf{P}\left(\frac{\operatorname{Cos} \psi}{\mathbf{P}_{1}} \overrightarrow{\mathbf{B}_{1}}+\frac{\operatorname{Sin} \psi}{\mathbf{P}_{2}} \overrightarrow{\mathbf{B}_{2}}\right)+\frac{\mathrm{d} \psi}{\mathrm{dS}} \overrightarrow{\mathbf{R}}_{0}
$$

bağıntısı elde edildi. Bu bağıntı kullanılarak yüzeyler teorisine ait Liouville, J. formülü, regle yüzeyin invaryantı olan dual küresel eğrilikler arasında bir bağıntı verilerek doğrular uzayına genelleştirildi ve bu bağıntılarla ilgili bazı sonuçlar verildi.

\section{REFERENCES}

[1] ÇALIŞKAN, A., On the Studying of a line Congruence by Choosing Parameter Ruled Surfaces as Principal Ruled Surfaces. Journal of faculty of Science of Fige University Seri A, P.23-33, 1987.

[2] BLASCHKE, W., Çeviri: Erim, K., Diferensiyel Geometri Dersleri, İst. Üniv. No: 443, IST., 1949.

[3] ERİM, K., Die höheren Differentialelemente einer Regelflaeche and einer Regelflaeche and einer Raumkurve, RFSUİ, S.A., T.X., Fasc. 1-4, İstanbul, 1946.

[4] BIIRAN, L., Mouvement à̀ un parametre, RFSUİ, S.A.T. XII., 208-229, İstanbul, 1947.

[5] BERKER, R., Mekanik Dersleri I, İst. Üni. Kütüphanesi, İstanbul, 1959.

[6] CARMO, M.P., Differential Geometry of Curves and Surfaces, Prentice Hall, inc., Englewood Cliffs, New Jersey, 1976. 